\title{
SPRING-DASHPOT MODELS FOR THE DYNAMICS OF A RADIALLY ROTATING BEAM WITH IMPACT
}

\author{
A. S. Yigit, A. G. Ulsoy and R. A. Scott \\ Department of Mechanical Engineering and Applied Mechanics, The University of Michigan, \\ Ann Arbor, Michigan 48109-2125, U.S.A.
}

(Received 17 October 1988, and in revised form 9 January 1990)

\begin{abstract}
In a previous study, experimental results for the dynamics of a radially rotating beam with impact were found to be in excellent agreement with simulation results using the momentum balance method (for impact modeling). In this paper spring-dashpot models for impact modeling are compared to experiment for a radially rotating flexible beam. Excellent agreement is found between the simulation results using spring-dashpot models and the experiments. A sensitivity study is employed to investigate the issue of accurately determining the model parameters.
\end{abstract}

\section{INTRODUCTION}

The impact of a flexible radially rotating beam against a rigid impact surface is considered (see Figure 1). In an earlier study [1] experimental results were compared with simulation results obtained by using the momentum balance (coefficient of restitution) model for impact. Although that model is not intended for application to systems with flexible members, good agreement was found between the experiments and the simulation. Sensitivity studies were employed to show that the model is applicable over a fairly wide range of parameter values. Thus, the momentum balance method has been demonstrated to be capable of accurately predicting the dynamics of systems which consist of both rigid and elastic links undergoing impact. A second competing method for impact modeling, which is applicable to flexible systems, is the spring-dashpot model. In this paper the validity and utility of spring-dashpot models are investigated for the dynamics of a radially rotating beam with impact.

A brief literature review on the spring-dashpot models for impact will now be given. Some of the energy losses during impact are associated with relative indentation and the damping mechanisms involved during this contact pcriod. The first attempt to incorporate a theory of local indentations is an elastostatic one given by Hertz [2]. The deformation is assumed to be restricted to the vicinity of the contact area and to be given by static theory. Elastic wave motion in the impacting bodies is neglected, and the total mass of each of the bodies is assumed to be moving at any instant with the velocity of its center of mass. The impact, therefore, can be visualized as the collision of two rigid bodies restricted to move in the direction of impact with spring buffers; all deformations occur in the springs, the inertias of which are neglected [3]. The assumption that deformation is quasi-static can only be justified if the duration of impact is long enough to permit the stress waves to tranverse the length of the structure many times [4]. This criterion (Love's criterion) does not apply in cases where an object impacts with another very large object, in which case no reflected wave returns to the point of impact [3]. Hunter [3] suggested as an alternative that the behavior of a large structure can be approximated by a dashpot in parallel with the spring to account for the energy radiated through the half-space by wave motion. If the time constant of the spring-dashpot system is short compared with 
the duration of the contact force, then the force variation during impact is determined predominantly by the spring; i.e., in a quasi-static manner. In this case the energy absorbed by the dashpot will be very small. Though less restrictive than Love's criterion, this criterion limits the velocity of impact to very low values [3]. Another limitation is that the Hertzian theory can be extended to describe only non-elastic impact conditions in which plastic flow extends to the vicinity of the contact point and does not permeate throughout the entire cross-section [2]. In view of these problems, there have been some attempts to determine a dynamic contact law based on some experimental measurements [5].

Several workers, notably Dubowsky and Freudenstein [6], have developed the so-called "impact-pair" model. They assumed a linear viscous damping law and a Hertzian spring for modeling the behavior of the impact surfaces. Dubowsky and Young [7] provided some experimental support for the impact-pair model. Hunt and Crossley [8], followed by Herbert and Mansour [9], argued that the damping coefficient in the case of vibroimpact should be proportional to a power $n$ of the spring force, and they adopted $n=1$ for analytical convenience. The advantages of this modification are a better representation of the variation of the energy loss with the approach velocity and a more realistic frequency content in the impulse generated [8]. Their results were in good agreement with experimental results on spheres and plates [10].

The character of impact between beam-like bodies has long been known to differ from that of impact between compact bodies. Dubowsky and Gardner [11] took the flexibilities of mechanism links into account and developed their "impact-beam" model. They showed that the dynamic behavior changes significantly due to the flexibility of the links [11].

In this paper spring-dashpot models are used having a Hertzian spring and the damping models developed by Lee and Wang [12]. Simulation results obtained by using the spring-dashpot models and the results of the experiments described in reference [1] are compared. Sensitivity studies are also employed to ascertain the generality of the results of these comparisons.

\section{SPRING-DASHPOT MODELS}

\subsection{STIFFNESS MODELS $[2,5,12]$}

A non-linear force-displacement law is the most widely used stiffness model for impact,

$$
F=k\left(x_{c}\right)^{n}
$$

where $F$ is the contact force, $x_{c}$ is the spring deflection caused by the impact of the rigid (assumed) body,

$$
n=3 / 2 \quad \text { and } \quad k=(4 / 3)\left\{q_{k} /\left(Q_{1}+Q_{2} \sqrt{(\overline{A+B})}\right\}\right.
$$

where $q_{k}, A$ and $B$ are given as functions of the geometry of the contacting bodies and,

$$
Q_{1}=\left(1-\mu_{1}^{2}\right) / E_{1} \pi, \quad Q_{2}=\left(1-\mu_{2}^{2}\right) / E_{2} \pi,
$$

in which $\mu_{i}$ and $E_{i}$ are the Poisson ratio and Young's modulus for the $i$ th body respectively. Note that $k$ and $n$ may also be obtained directly from experiments.

\subsection{DAMPING MODELS $[2,3,6,11,12]$}

We consider two damping models proposed by Lee and Wang [12]. For both the damping force is of the form

$$
f_{d}=C T\left(x_{c}\right) \dot{x}_{c}
$$


In their first damping model they used the damping function

$$
T_{1}\left(x_{c}\right)=x_{c},
$$

and expressed the coefficient of restitution in terms of the impact velocity $\nu_{i}$ by

$$
e=\alpha_{0}-\sum_{n=1}^{x} \alpha_{n} \nu_{l}^{\prime \prime}
$$

The $\alpha_{n} s$, determined by a least-squares fit to experimental data, are assumed to be material properties. Then they solved approximately the non-linear one-dimensional problem of a point mass $M$ contacting a surface through a linear spring (constant $k$ ) and a dashpot, the constitutive law of which is the same as equation (6). They calculated the coefficient of restitution $e$ in terms of $k$ and $C$, and equated this with the experimental least-squares fit to obtain the damping coefficient $C\left(\equiv C_{1}\right)$ as a function of $k$ and $\alpha_{1}$. They used only the first two terms in the series for $e$, and showed that the damping coefficient for their first model $C_{1}$ turns out approximately to be

$$
C_{1}=0.75 \alpha_{1} k
$$

An advantage of this model is that the damping coefficient is calculated as a function of $\alpha_{1}$, which can be thought of as a material property, independent of velocity.

Lee and Wang [12] pointed out, however, that this first damping model may cause instability due to its non-linear characteristic. To overcome such shortcomings, they developed a second damping model,

$$
f_{d}=C_{2} T_{2}\left(x_{c}\right) \dot{x}_{c},
$$

where the damping function is

$$
T_{2}\left(x_{c}\right)=\left[\left(x_{c}+\left|x_{c}\right|\right) / 2 x_{c}\right] \exp \left[\left\{\left(x_{c}-\varepsilon\right)-\left|x_{c}-\varepsilon\right|\right\}(Q / \varepsilon)\right],
$$

where $\varepsilon$ defines a so-called transition zone, and $Q$ is a parameter specifying the shape of the curve within the transition zone. The damping function in equation (11) is zero when $x_{c} \leqslant 0 \cdot 0$, and is one when $x_{c} \geqslant \varepsilon$. The value of $\varepsilon$ may be arbitrarily chosen, but it must satisfy the conditions [12]

$$
0 \leqslant \varepsilon \leqslant x_{c \max }, \quad C_{2} T_{2}\left(x_{c}\right) \dot{x}_{c}+k\left(x_{c}\right)^{3 / 2} \geqslant 0 .
$$

Conditions (12) and (13) assure the positiveness of the sum of the damping and spring forces. Then, $C_{2}$ is calculated by using the same procedure as for $C_{1}$, but with attention restricted to the post transition phase, (for which $T_{2}=1$ ). The result is

$$
C_{2}=2 M \omega_{n} \sqrt{(\ln e)^{2} /\left[(\ln e)^{2}+\pi^{2}\right]},
$$

where $e$ is the coefficient of restitution appropriate for the initial impact velocity [2], and $\omega_{n}=\sqrt{k / M}$.

The main advantage of this second damping model is that it is linear after the transition zone. The number of parameters $\left(Q, \varepsilon, C_{2}\right)$ which have to be specified for the model however, is greater than for the first damping model. It was shown in references $[1,14]$ that multiple impacts can occur in flexible systems. This raises the question whether one should update the $C_{2}$ value for each of these multiple impacts, since they occur at different velocities. The sensitivity studies presented later in this paper will show the model is not sensitive to the value of $C_{2}$ within the practical range of coefficient of restitution values. Thus an updating procedure for $C_{2}$ is not necessary. 


\section{SIMULATION RESULTS AND COMPARISONS}

Both damping models presented in the previous section have been studied for the system in Figure 1. A sensitivity study was also performed to investigate the effect of the damping coefficient on the dynamic behavior. For the rotating beam (see Figure 1)

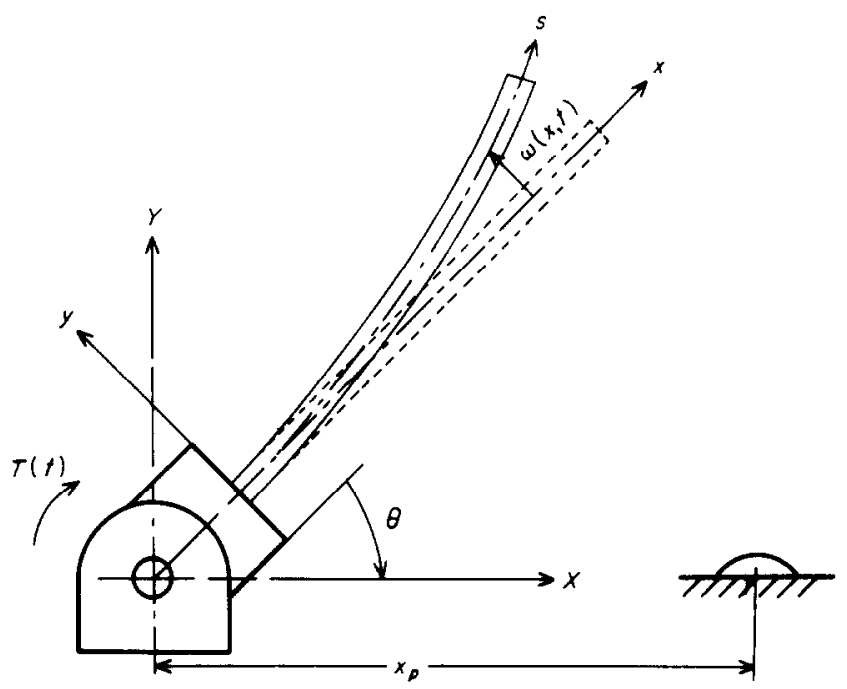

Figure 1. The sketch of the rotating beam.

discretized equations of motion, including an impact force $F(t)$, have been derived in reference [13] by using Galerkin's method:

$$
\sum_{j=1}^{N}\left\{\ddot{q}_{j}\left(m_{r j}\right)+\dot{\theta}^{2} q_{j}\left(c_{r j}-m_{r j}\right)+q_{j} k_{r j}\right\}+S_{r} \ddot{\theta}+\sum_{j=1}^{N} \rho g S_{j}^{*} \cos \theta=\phi_{r}\left(x_{r}\right) F(t),
$$

where $r=1,2, \ldots, N$, and

$$
\left(I_{R}+I_{B}\right) \ddot{\theta}+\sum_{j=1}^{N} \ddot{q}_{j} S_{j}+\frac{1}{2} \rho g l^{2} c \cos \theta-\sum_{j=1}^{N} S_{j}^{*} q_{j} g \sin \theta=T(t)+x_{p} F(t) .
$$

The number of modes used in the simulation is $N$, and

$$
\begin{gathered}
m_{r j}=\int_{0}^{l} \rho \phi_{r} \phi_{j} \mathrm{~d} x, \quad c_{r j}=-\int_{0}^{l}\left[\frac{1}{2} \rho\left(l^{2}-x^{2}+2 a l-2 a x\right) \phi_{r}^{\prime \prime} \phi_{j}+\rho(x+a) \phi_{r}^{\prime} \phi_{j}\right] \mathrm{d} x, \\
k_{r j}=\int_{0}^{l} E I \phi_{r}^{\prime \prime \prime \prime} \phi_{j} \mathrm{~d} x, \quad S_{r}=\int_{0}^{l} \rho(x+a) \phi_{r} \mathrm{~d} x, \quad S_{r}^{*}=\int_{0}^{l} \rho \phi_{r} \mathrm{~d} x .
\end{gathered}
$$

Here $l$ is the length of the beam, $\alpha$ is the radius of the rigid shaft, $I_{R}$ is the moment of inertia of the rigid shaft about the axis of rotation, $I_{B}$ is the moment of inertia of the flexible beam, and $\rho$ is the mass per unit length. $E I$ denotes the flexural rigidity of the beam, and $g$ is the acceleration of gravity. The $q_{i}(t)$ are the unknown generalized coordinates and the $\phi_{i}(x)$ are a set of comparison functions that satisfy all the boundary conditions. Here they are chosen as the mode shapes for the non-rotating cantilever beam. The position along the beam at which impact occurs is denoted by $x_{p}$, and $F(t)$ is given by

$$
F(t)=\left\{\begin{array}{ll}
F_{1}(t) & \text { if } F_{1}(t)>0 \\
0 & \text { if } F_{1}(t) \leqslant 0
\end{array}\right\}
$$


where

$$
F_{1}(t)=k\left(x_{c}\right)^{n}+C T\left(x_{c}\right) \dot{x}_{c} .
$$

Parameters for the two spring-dashpot models described in the previous section were selected to be $[2,14] A=1 / 2 R_{1}, B=1 / 2 R_{2}, R_{1}=0.0063 \mathrm{~m}, R_{2}=0.0126 \mathrm{~m}, E_{1}=E_{2}=$ $6.895 \times 10^{10} \mathrm{~N} / \mathrm{m}^{2}, \quad \mu_{1}=\mu_{2}=0.3, Q=3.0, \quad \varepsilon=0.5 \times 10^{-5} \mathrm{~m}, \quad k=3.2 \times 10^{9} \mathrm{~N} / \mathrm{m}, \quad C_{1}=$ $1.92 \times 10^{8} \mathrm{~kg} / \mathrm{ms}$, and $C_{2}=800 \mathrm{~kg} / \mathrm{s}$.

Condition (13) is checked during the contact phase of the simulation to assure the positiveness of the sum of the spring and damping forces. Condition (12) is checked after the simulation is completed to ensure that the selection of $\varepsilon$ is realistic. Contact force is calculated and monitored. Whenever it becomes negative this represents the separation of the objects and the contact force is set to be zero so that there is no tension in the spring.

In order to achieve a smooth response, the time step for the integration should be adjusted to catch the initial contact with a minimum of spring deflection. A successful procedure was found to be as follows [15]. The distance between the impact point and the beam was monitored during the simulation, and whenever it became smaller than $10 \varepsilon, \varepsilon$ being the length of the transition zone, the integration time step $\Delta t$ was reduced to $(1 / 100) \Delta t_{1}$, where $\Delta t_{1}$ is the original time step before impact. The reduced time step is maintained until the beam separates from the impact surface by the same amount (i.e., $10 \varepsilon$ ). With this algorithm it is possible to capture multiple impacts.

For the simulations presented here a three mode approximation was used. Simulations involving five and seven modes were also performed and no perceptible difference was found with respect to the dynamic behavior. Thus three modes are considered adequate for accurately describing the elastic motion for the simulations reported in this paper.

The measured torque input, shown in Figure 2, was approximated by a pulse in the simulations. Simulation result for the angular velocity of the rigid stem are shown in Figures 3 and 4 for the two damping models. The corresponding experimental result which was reported in reference [14] is shown in Figure 5. Strain histories at one location along the beam $(x=0.275 \mathrm{~m})$ are shown in Figures 6 and 7 , again for the two damping models. The experimental result for the strain history at the same location is shown in Figure 8 .

Comparing the simulation results for the two damping models, one can conclude that both models have almost identical performances with regard to capturing the dynamics of the system after impact. In the results from the first damping model a third set of

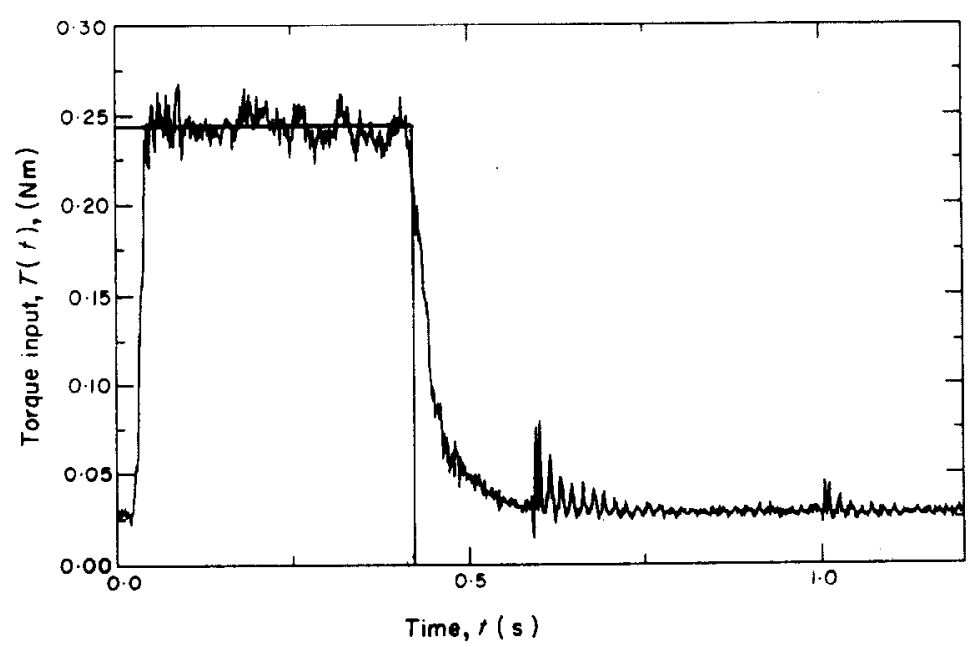

Figure 2. Measured torque input, and approximated torque pulse for simulation. 


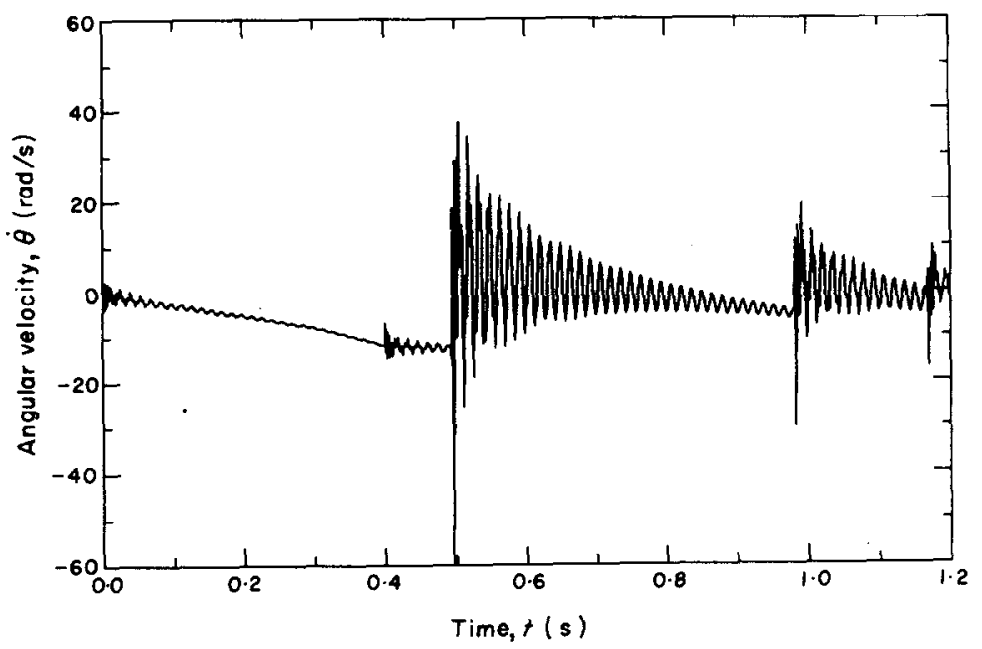

Figure 3. Angular velocity of the rigid shaft, first damping model.

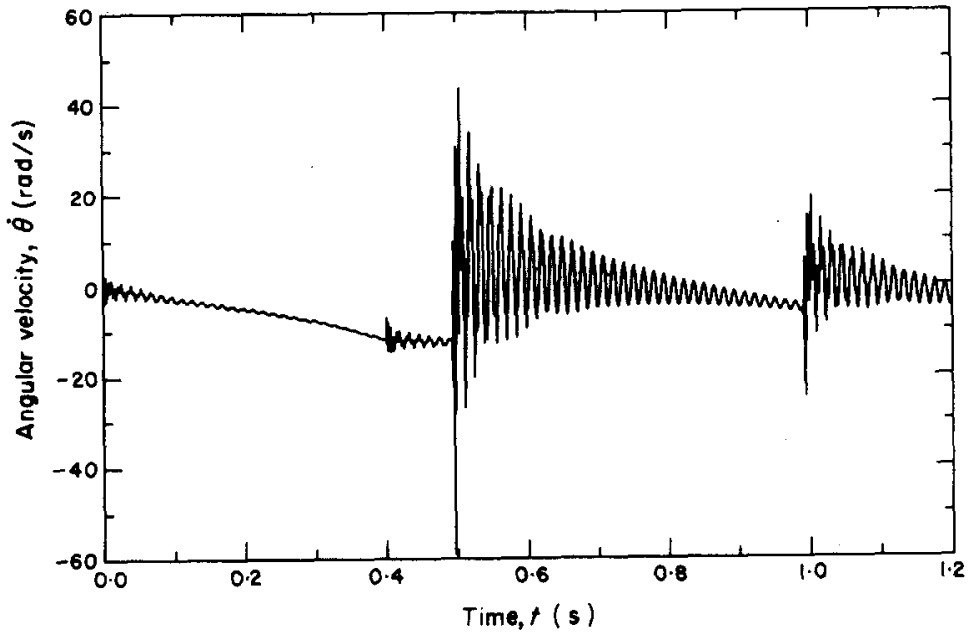

Figure 4. Angular velocity of the rigid shaft, second damping model.

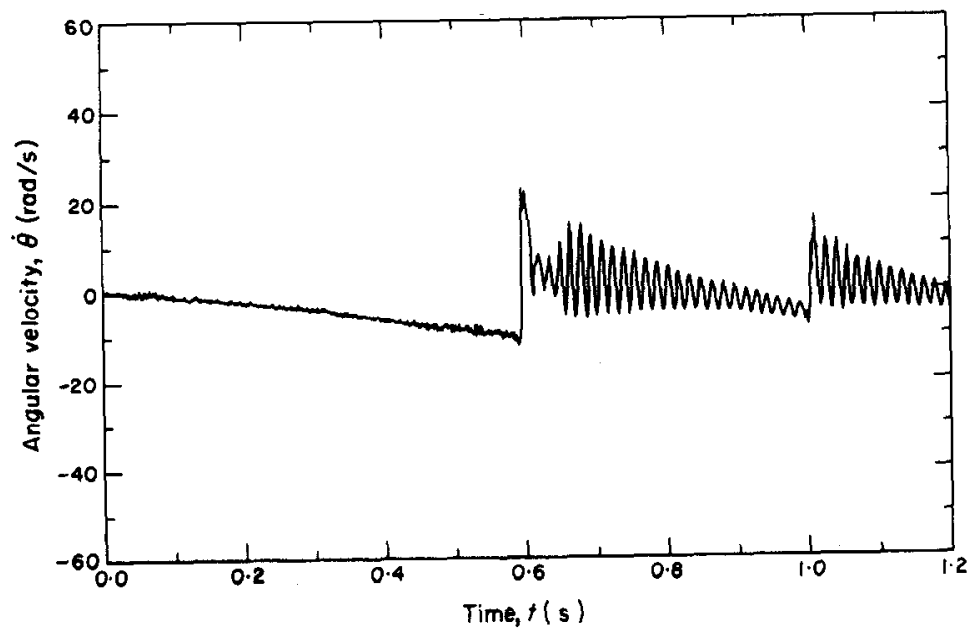

Figure 5. Angular velocity of the rigid shaft, experiment. 


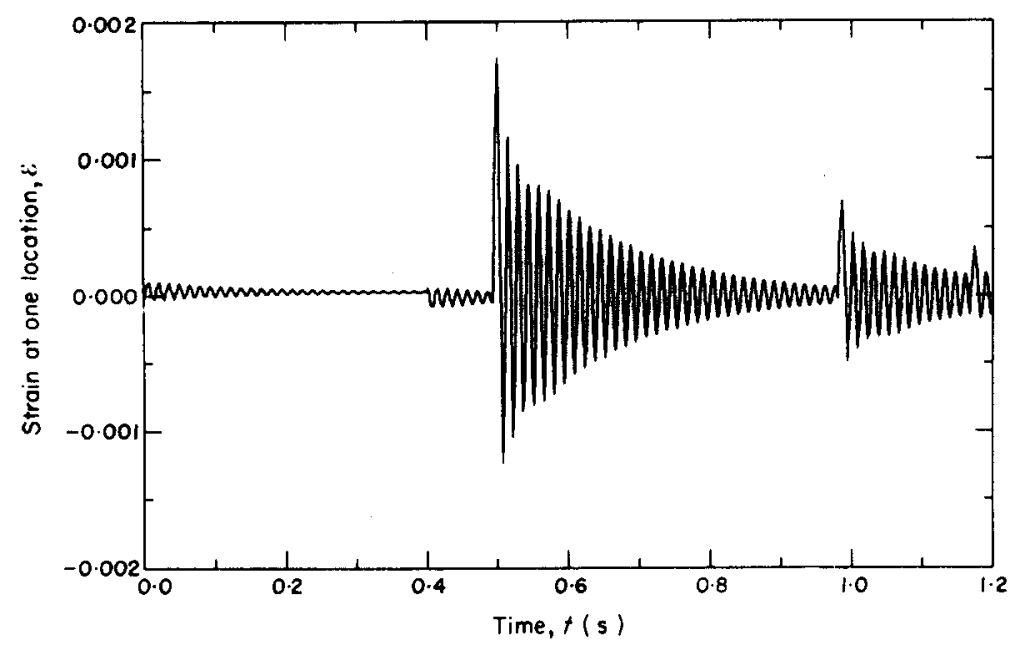

Figure 6. Strain history at one location, first damping model.

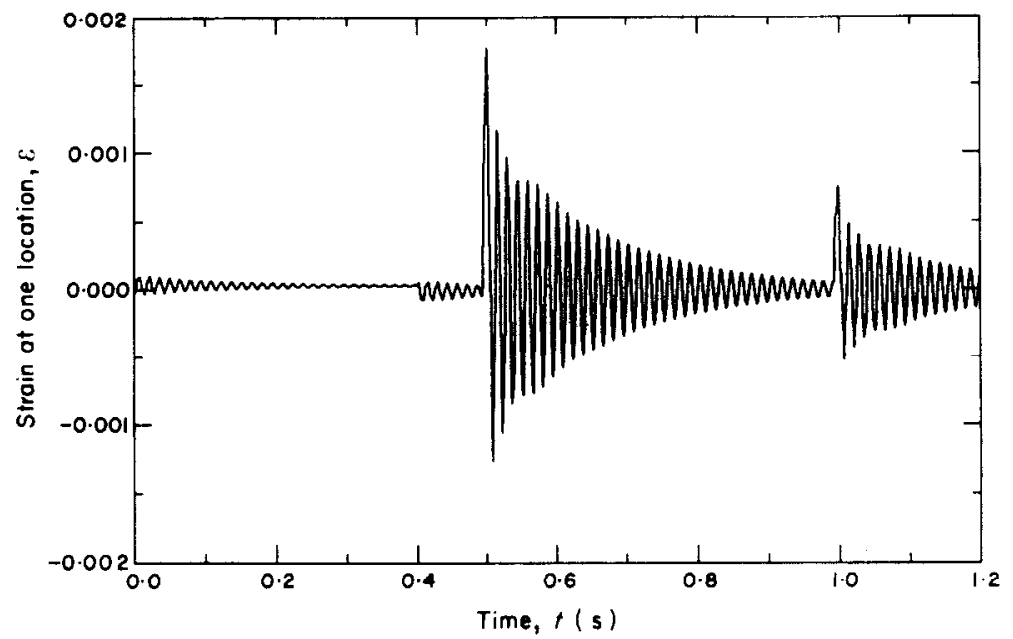

Figure 7. Strain history at one location, second damping model.

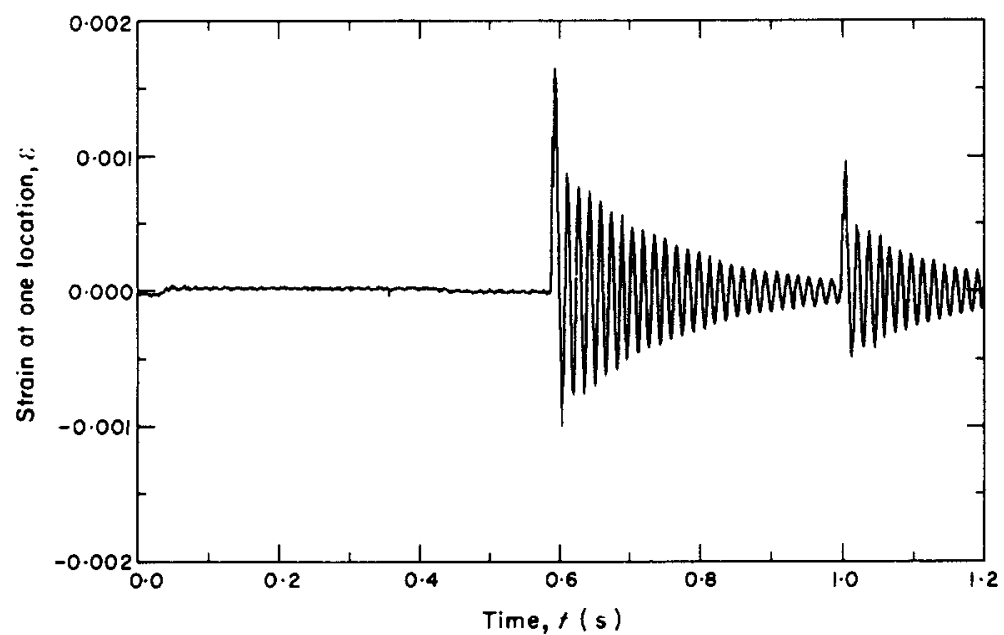

Figure 8 . Strain history at one location, experiment. 
impacts is seen to have occurred at approximately $t=1 \cdot 15 \mathrm{~s}$ (see Figure 6). However, this impact is not seen in Figure 8 for the second damping model. The reason is that the two models do not have exactly the same amount of energy dissipation; thus the effective coefficient of restitution values for the first set of impacts turns out to be different. The third set of impacts would, thus, occur at a different time for the second damping model.

The dominant frequency predicted by the simulations is about $67 \mathrm{~Hz}$, and this agrees well with the experimental result of $66 \mathrm{~Hz}$ (Figure 8). Peak strains also agree well as can be seen by comparing Figures 8 and 6 , or 7 . Due to the presence of Coulomb friction at the joint (not modelled in the simulation) there is a time delay in the experimental results. This effect can be observed by comparing Figures 5 and 3, or 4 . The effect of Coulomb friction is seen only at the beginning of the motion when the angular velocity is small, and it diminishes as the angular velocity increases. The angular velocity obtained from the simulation shows a much higher negative peak at the time of initial impact than the one observed in the experiment. This difference is probably due to the fact that the angular acceleration for this region is beyond the limit of the incremental optical encoder which was used to measure the angular velocity. The average acceleration at the time of impact as calculated from the simulation is $110000\left[\mathrm{rad} / \mathrm{s}^{2}\right]$. The negative peaks for the subsequent impacts are captured accurately, and they do fall within the acceleration limit of the encoder.

In summary, it can be concluded that both damping models, with appropriately evaluated parameters, give excellent agreement with the experimental results reported in [14].

\section{SENSITIVITY STUDIES FOR THE SPRING-DASHPOT MODEL}

To give the above findings greater generality, some sensitivity studies will now be presented. There are many possible measures of sensitivity. Here two sensitivity measures employed previously in [1] are investigated:

1. Peak elastic deflection sensitivity, $s_{1}$. This is basically a normalized peak deflection, and can be written as

$$
s_{1}=\left[w(l, t)_{\max }-\bar{w}(l, t)_{\max }\right] / \bar{w}(l, t)_{\max },
$$

where $w(l, t)_{\max }$ are peak elastic deflections calculated for different values of $e$, and $\bar{w}$ $(l, t)_{\max }$ is the arithmetic mean of all those $w(l, t)_{\max }$ values.

2. Tip-displacement sensitivity, $s_{2}$. Another possible measure for sensitivity is the total displacement $w_{p}$ due to both elastic and rigid-body motion. Because of the rigid-body motion, $w_{p}$ does not have a maximum value, and so replacing $w$ by $w_{p}$ in equation (24) is not appropriate. A sensitivity measure $s_{2}$ (which is a function of time) is introduced by

$$
s_{2}=\left[w_{p}(l, t)_{c_{2}=500}-w_{p}(l, t)\right] / w_{p}\left(l, t_{1}\right)_{c_{2}=500},
$$

where $w_{p}(l, t)$ is the displacement corresponding to an arbitrary value of $C_{2}$, and $w_{p}(l, t)_{c_{2}=500}$ is the tip displacement for $C_{2}=500$. The value 500 is selected arbitrarily as an intermediate value of $C_{2}$ in the range of practical values, and corresponds to a coefficient of restitution value of 0.46 . The symbol $t_{1}$ denotes a time after impact. In the sensitivity plots presented $t_{1}$ is taken as the final time in the simulations. For the results presented here $t_{1}=0.2 \mathrm{~s}$, and a torque pulse of $1 \mathrm{Nm}$ is applied to the rigid shaft for a duration of $0.05 \mathrm{~s}$.

Values of $s_{1}$ for two different inertia ratios $\left(=I_{R} / I_{B}\right)$ are shown in Figure 9 . In both cases elastic peak deflections vary only by about 5 percent for a wide range of damping 


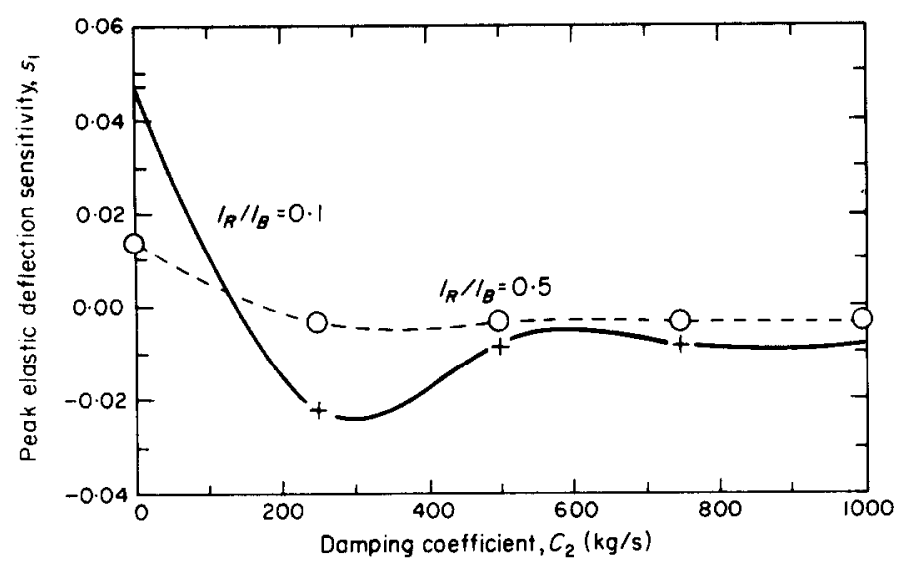

Figure 9. Peak elastic tip-deflection sensitivity.

coefficient values in the practical range of 0 to $1000 \mathrm{~kg} / \mathrm{s}$ corresponding to coefficient of restitution values of 0.4 to 0.8 .

Results for the $s_{2}$ sensitivity measure are presented in Figure 10 for different values of $C_{2}$ and one value of flexural rigidity. In all cases the maximum value is about 6 percent up to the final time in the simulation. Similar small values for $s_{2}$ were found for other rigidity values.

All the above sensitivity results suggest that a damping coefficient value which is appropriately chosen from the rigid-body coefficient of restitution data would give sufficiently accurate results, within engineering tolerances, for many situations of practical interest. By appropriately chosen we mean that it should be selected based on the expected initial impact velocity, and the material pair in question.

\section{SUMMARY AND CONCLUSIONS}

Spring-dashpot models were presented to study the dynamics of a radially rotating beam with impact. The models use the Hertzian contact law and damping models, and enable one to predict the rigid-body motion as well as the elastic motion during, and after impact. Two different damping models proposed by Lee and Wang [12] were implemented and compared for the specific problem of the radially rotating beam with

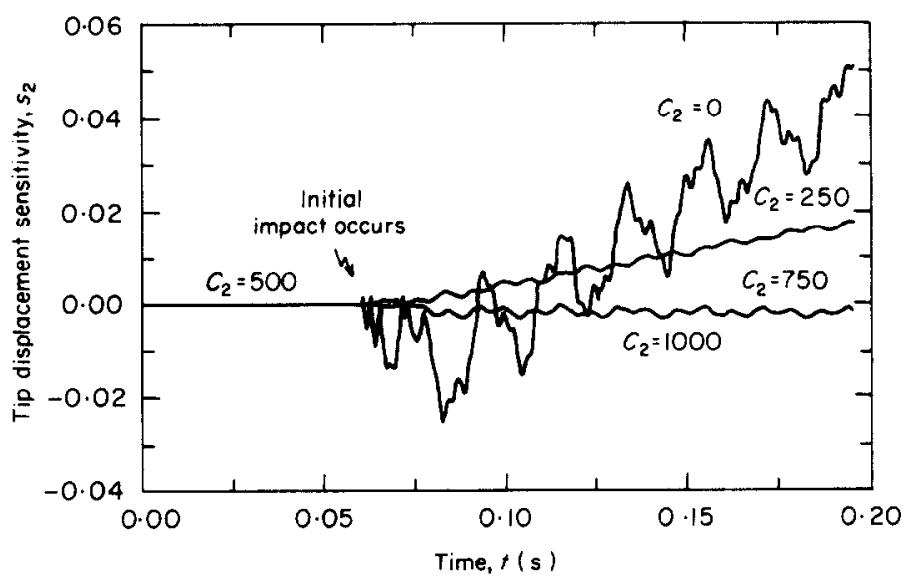

Figure 10. Total tip-deflection sensitivity for $E I=5 \cdot 50$, 
impact. They were found to give very similar results, which compared well with the experimental results reported previously in reference [14]. A sensitivity study was performed to investigate the importance of the value of the damping coefficient for the second damping model. It was found that the dynamic behavior of the system is not sensitive to the value of the damping coefficient over a wide range which covers most practical situations.

Compared to the momentum balance method described previously in reference [1], the spring-dashpot models reported here require additional parameters that have to be determined. However, they have the merit of giving the contact force. Also it was found from the sensitivity studies that the models do not require a very accurate determination of the damping coefficient. Computationally all models, including the momentum balance model reported previously in reference [1], were found to be roughly equivalent for the specific problem treated here. However, the computational efficiency is a problem dependent issue. In the momentum balance method a set of linear algebraic equations, the number of which is dependent on the degree of freedom of the system, has to be solved for each impact [1]. On the other hand it does not require integration (at a reduced step size) over the contact duration. Spring-dashpot models do not require the solution of linear algebraic equations, but rather the integration of the equations of motion for the contact duration. For a system consisting of rigid bodies the momentum balance method has a clear computational advantage over the spring-dashpot models. However, for systems involving flexible members the issues is not clear cut, and depends on the rigid and elastic degrees of freedom of the system.

\section{REFERENCES}

1. A. YIGIT, R. A. SCOTT and A. G. Ulsoy 1990 American Society of Mechanical Engineers Journal of Vibration Acoustics, Stress and Reliability in Design 112,65-70. Dynamics of a radially rotating beam with impact, Part 1: theoretical and computational model.

2. W. GoldSMITH 1960 Impact. London: Edward Arnold.

3. K. L. JOHNSON 1985 Contact Mechanics. Cambridge University Press.

4. A. E. LOVE 1944 A Treatise on the Mathematical Theory of Elasticity. New York: Dover.

5. J. F. DOYLE 1987 Experimental Mechanics, 27, 68-72. Determining the contact force during the transverse impact of plates.

6. S. DUBOWSKY and F. FREUDENSTEIN 1971 American Society of Mechanical Engineers Journal of Engineering for Industry 93, 305-316. Dynamic analysis of mechanical systems with clearances, Part 1: formation of dynamic model.

7. S. DUbowSKY and S. C. YoUNG 1975 American Society of Mechanical Engineers Journal of Engineering for Indusiry 97B, 1166-1174. An experimental and analytical study of connection forces in high-speed mechanisms.

8. K. H. HUNT and F. R. E. CROSSLEY 1975 American Society of Mechanical Engineers Journal of Applied Mechanics 42E, 440-445. Coefficient of restitution interpreted as damping in vibroimpact.

9. R. G. HERBERT and W. M. MANSOUR 1977 American Society of Mechanical Engineers Journal of Engineering for Industry, 98B, 513-518. Shape and frequency composition of pulses from an impact pair.

10. M. A. Veluswami and F. R. E. Crossley 1977 American Society of Mechanical Engineers Journal of Engineering for Industry, 97B, 820-827. Multiple impacts of a ball between two plates.

11. S. DUBOWSKY and T. N. GARDNER 1975 American Society of Mechanical Engineers Journal of Engineering for Industry 97B, 652-661. Dynamic interactions of link elasticity and clearance connections in planar mechanical systems.

12. T. W. LEE and A. C. WANG 1983 American Society of Mechanical Engineers Journal of Mechanisms, Transmissions, and Automation in Design 105, 534-540. On the dynamics of intermittent-motion mechanisms, Part 1: dynamic model and response.

13. A. YIGIT, R. A. SCOTt and A. G. Ulsoy 1988 Journal of Sound and Vibration 121, 201-210. Flexural motion of a radially rotating beam attached to a rigid shaft. 
14. A. Yigit, A. G. UlsoY and R. A. SCOTT 1990 American Society of Mechanical Engineers Journal of Vibration, Acoustics, Strees and Reliability in Design 112, 71-77. Dynamics of a radially rotating beam with impact, Part 2: experimental and simulation results.

15. A. YIGIT 1988 Ph.D. Dissertation, The University of Michigan, Ann Arbor, Michigan. Dynamics of a Radially Rotating Beam with Impact: Implications for Robotics. 